\title{
is Research Square \\ Infection With The Pinworm Blatticola Blattae And Survival of Starving German Cockroaches, Blattella Germanica
}

Masuko Kobayashi

Azabu University

Kensuke Taira ( $\boldsymbol{\nabla}$ taira@azabu-u.ac.jp )

Azabu University

\section{Research Article}

Keywords: Pinworm infection, survival, German cockroach

Posted Date: August 6th, 2021

DOl: https://doi.org/10.21203/rs.3.rs-782833/v1

License: (c) (1) This work is licensed under a Creative Commons Attribution 4.0 International License.

Read Full License 


\section{Abstract}

The German cockroach, Blattella germanica, is often infected with the pinworm, Blatticola blattae. To investigate the effect of pinworm infection on cockroach survival, we artificially manipulated the pinworm infection status of two lines of German cockroaches kept in the laboratory and compared survival rates in starvation. Of the two lines of German cockroaches WATn and NIIDp bred in the laboratory, the WATn line was not infected with pinworm, and the NIIDp line was naturally infected with pinworm B. blattae. The WATp line was created by artificially infecting the pinworm B. blattae with this WATn line. The NIIDn line was created by treating the NIIDp line with anthelmintic drugs. The $50 \%$ survival days of uninfected WATn line of starving cockroaches was 8 days, and infected WATp line of starving cockroaches was 13 days. The $50 \%$ survival days of naturally infected NIIDp line of starving cockroaches was 6 days, and that of anthelmintic-treated NIIDn line of cockroaches was 4 days. The survival days were significantly longer in pinworm-infected cockroaches than uninfected or anthelmintic-treated cockroaches. These results suggest that pinworm infection enhances the survival of cockroaches under starving conditions.

\section{Introduction}

The German cockroach, Blattella germanica, is frequently infected with the pinworm, Blatticola blattae (Kobayashi et al. 2021). Although pinworm infection is common among cockroaches worldwide, few reports have described the effects of parasite infection on the insect host. In order to investigate the effects of nematode infection in cockroaches, it is necessary to control the pinworm infection. Kobayashi et al. (2021) reported that German cockroaches can be easily infected with B. blattae via contact with feces from pinworm-infected cockroaches. Anthelmintics of pyrantel pamoate and pyrvinium pamoate were reported to be effective for deworming B. blattae in the German cockroaches, without causing mortality for the host (Kobayashi et al. 2020). Based on these reports, we established a line of German cockroaches that originated from a pinworm-free line and artificially infected the cockroaches with pinworms. We also established an anthelmintic-treated line of German cockroaches that originated from a line naturally infected with pinworms.

The purpose of this study was to investigate the effect of $B$. blattae infection on the survival of German cockroaches under starvation conditions. We compared the number of days until the cockroach survival rate reached $50 \%$ for cockroaches that artificially changed the pinworm infection status.

\section{Materials And Methods}

\section{German cockroach}

Four lines of German cockroach (WATn, WATp, NIIDn, and NIIDp) were used in the study. WATn is originally a pinworm-uninfected WATARIDA strain, and is abbreviated as WATn by adding " $n$ " which means pinworm-negative. WATp is a cockroach strain that artificially infected the WATARIDA strain with B. blattae, and is designated as WATp by adding " $p$ ", which means pinworm- positive. WATn cockroaches 
were artificially infected with $B$. blattae by exposing them to feces of infected cockroaches for 6 months in the breeding environment. The prevalence of $B$. blattae infection was $0 \%(0 / 40)$ in the WATn line and $83 \%(20 / 24)$ in the WATp line.

NIIDp is naturally pinworm-infected NIID strain, with "p" meaning pinworm-positive. The NIIDn strain was artificially generated by treating pinworm-infected NIIDp line with an anthelmintic (2000 ppm pyruvinium pamoate solution) for 16 days, and the negative meaning " $n$ " was added to make NIIDn. The prevalence of B. blattae was $93 \%$ (40/43) for the NIIDp line and 6.7\% (1/15) for the NIIDn line.

Adult German cockroaches 3 to 30 days old for the WATp and WATn lines and 24 to 50 days old for the NIIDp and NIIDn lines were used in the study.

\section{Cockroach breeding environment}

A total of 10 to 15 adult German cockroaches were bred in a plastic cage (size: $175 \times 140 \times 105 \mathrm{~mm}$ ) with a porous cover to allow air entry. Escape of cockroaches was prevented by applying a 50-mm wide layer of Vaseline (manufactured by Kanto Chemical Co., Inc.) to the upper surface of the inner wall of the container. A piece of folded paper (approx. $70 \times 70 \mathrm{~mm}$ in size) was placed in the cage to provide a hiding place. Water and food (control area only) were also provided, and the cages were kept in a constant temperature environment $\left(25 \pm 1^{\circ} \mathrm{C}\right)$.

\section{Comparison of survival rate}

Pinworm-infected cockroaches (WATp \& NIIDp), uninfected cockroaches (WATn), and anthelmintic-treated cockroaches (NIIDn) were bred separately in two groups, a normal feeding group and a non-feeding group. The number of surviving cockroaches was recorded at the end of each day. Feces excreted by the cockroaches during the test period were not removed until the end of the experiment.

\section{Statistical analysis}

For each group, the $50 \%$ survival days (the number of days until the survival rate reached $50 \%$ ) was determined from the survival rate in the elapsed days. The $50 \%$ survival days was calculated according to the Kaplan-Meier method and the log-rank test was used to examine the significance of the differences. The analysis was performed in a high-level test using Statcel 4 software (OMS Publishing, Tokyo, Japan) and a $P$ value of $\leq 0.05$ was considered significant.

\section{Results}

Table 1 summarizes the results of a comparison of the number of surviving cockroaches between the pinworm-infected lines (WATp and NIIDp) and uninfected line (WATn) or anthelmintic-treated line (NIIDn). WAT line was used in Experiment 1 and NIID line was used in Experiment 2. When fed a normal diet, 100\% of the cockroaches in both experiments survived to the end of the study period. In the absence of food, survival of WATn cockroaches reached $50 \%$ after 8 days, whereas 13 days were required for survival of 
WATp cockroaches to reach 50\%. In Exp. 2, survival of anthelmintic-treated NIIDn cockroaches declined to zero in 6 days, but NIIDp cockroaches continued to survive even after 10 days.

Table 1. Comparison of the number of surviving German cockroaches, Blattella germanica, between pinworm (Blatticola blattae)-infected lines and un-infected or anthelmintic-treated lines.

\begin{tabular}{|c|c|c|c|c|c|c|c|c|c|c|}
\hline & \multirow[t]{2}{*}{$\operatorname{Diet}^{1)}$} & \multirow[t]{2}{*}{ B. blattae } & \multirow{2}{*}{$\begin{array}{l}\text { Cockroach } \\
\text { line }^{2)}\end{array}$} & \multicolumn{7}{|c|}{ Days after treatment } \\
\hline & & & & 1 & 5 & 7 & 9 & 13 & 14 & 15 \\
\hline \multirow[t]{6}{*}{ Exp. 1} & Normal & Infected & WATp & 50 & 50 & 50 & 50 & 50 & 50 & 50 \\
\hline & & Free & WATn & 50 & 50 & 50 & 50 & 50 & 50 & 50 \\
\hline & None* & Infected & WATp & 53 & 53 & 50 & 41 & 23 & 10 & 6 \\
\hline & & Free & WATn & 51 & 47 & 32 & 10 & 0 & 0 & 0 \\
\hline & & & & \multicolumn{7}{|c|}{ Days after treatment } \\
\hline & & & & 1 & 4 & 6 & 7 & 10 & & \\
\hline \multirow[t]{4}{*}{ Exp. 2} & Normal & Infected & NIIDp & 15 & 15 & 15 & 15 & 15 & & \\
\hline & & Treated & NIIDn & 16 & 16 & 16 & 16 & 16 & & \\
\hline & None* & Infected & NIIDp & 15 & 10 & 6 & 5 & 1 & & \\
\hline & & Treated & NIIDn & 16 & 2 & 0 & 0 & 0 & & \\
\hline
\end{tabular}

1) For the normal diet, approximately $3 \mathrm{~g}$ of solid feed for mice (MF Oriental Yeast Co., Ltd., Tokyo, Japan) was given. For 'None', no feed was given.

2) The prevalence of B. blattae in the WATp and WATn lines was $83 \%(20 / 24)$ and $0 \%(0 / 40)$, respectively. WATp cockroaches were experimentally infected with pinworms via exposure to feces from pinworm-infected cockroaches in the breeding environment. The prevalence of B. blattae in the NIIDp and NIIDn lines was 93\% (40/43) and 6.7\% (1/15), respectively. The NIIDn group is a line in which the infection rate was reduced by treatment of pinworm-infected NIIDp roaches with an anthelmintic agent (pyrvinium pamoate 2000 ppm solution) for 16 days.

*WATp vs. WATn: $\mathrm{P}=3.89 \times 10^{-8}$, NIIDp vs. NIIDn: $\mathrm{P}=0.048$ (log-rank test)

Figure 1 shows cockroach survival curves for Exp. 1 and Exp. 2 without food. In Exp. 1, the 50\% survival period was 13 days for pinworm-infected WATp cockroaches and 8 days for uninfected WATn roaches. A significant difference in survival was noted between the WATp and WATn lines (log-rank test: $P=$ $3.89 \times 10^{-8}$ ). In Exp. 2, the $50 \%$ survival period was 6 days for pinworm-infected NIIDp cockroaches and 4 days for anthelmintic-treated NIIDn roaches. A significant difference in survival was also noted between the NIIDp and NIIDn lines (log-rank test: $P=0.048$ ). 


\section{Discussion}

The results of this study suggest that pinworm-infected cockroaches survive longer than uninfected cockroaches under starvation conditions. The mechanism underlying this difference will need to be investigated in further studies. We speculate that pinworm-infected cockroaches have higher tendency to engage in coprophagy (i.e., ingesting feces as a nutrient source), which consequently enables them to survive longer than uninfected cockroaches.

The host-parasite relationship between German cockroaches and $B$. blattae pinworms may represent a unique model for studies of relationship between parasitic nematodes and the hosts. Based on our results, we speculate that the German cockroach and $B$. blattae have a mutually beneficial relationship. The German cockroach is an important sanitary insect pest that is widely found in urban human living spaces worldwide. In addition, numerous studies have indicated that $B$. blattae is a common nematode parasite of the German cockroach (Adamson and Waerebeke 1992; Jarry 1964; Tsai and Cahill 1970; Kobayashi et al. 2021). The results of the present study suggest that $B$. blattae infection enhances the survival of German cockroaches.

In conclusion, the present study demonstrated for the first time that pinworm-infected German cockroaches can survive longer than uninfected cockroaches under starvation conditions. Our results suggest that the German cockroach and pinworm B. blattae have a mutually beneficial relationship.

\section{Declarations}

\section{Conflict interest}

The authors declare that they have no conflict of interest.

\section{References}

1. Adamson, M. L. \& Waerebeke, D. Revision of the Thelastomatoidea, Oxyurida of invertebrate hosts. I. Thelastomatidae. Syst Parasitol, 21, 21-63 https://doi.org/10.1007/BF00009911 (1992).

2. Jarry, D. T. Oxyurouidea of some arthropods in the south of France. Ann. Parasitol. Hum. Comp, 39, 381-508 https://doi.org/10.1051/parasite/1964394381 (1964).

3. Kobayashi, M., Ooi, H. K. \& Taira, K. (2020) Effects of anthelmintics on the pinworm Blatticola blattae in laboratory-reared German cockroaches Blattella germanica. Parasitology Research; 119(9):30933097. https://doi.org/10.1007/s00436-020-06778-1

4. Kobayashi, M., Komatsu, N., Ooi, H. K. \& Taira, K. Prevalence of Blatticola blattae (Thelastomatidae) in German cockroaches Blattella germanica in Japan. ã€€J. Vet. Med. Sci, 83 (2), 174-179 https://doi.org/10.1292/jvms.20-0617 (2021).

5. Tsai, Y. H. \& Cahill, K. M. Parasites of the German Cockroach (Blattella germanica L.) in New York City. J Parasitol, 56, 375-377 https://doi.org/10.2307/3277678 (1970). 
Figures

\section{Exp.1}

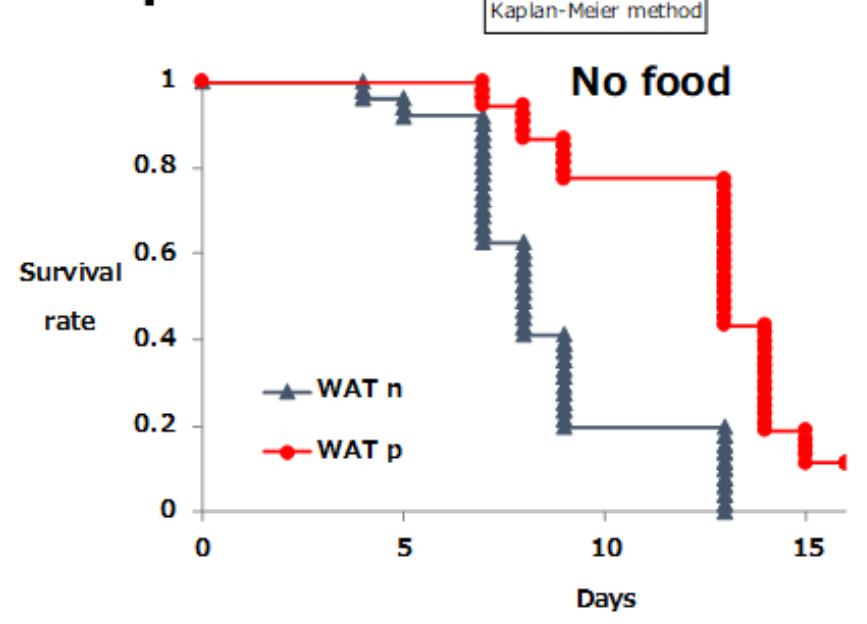

\section{Exp.2}

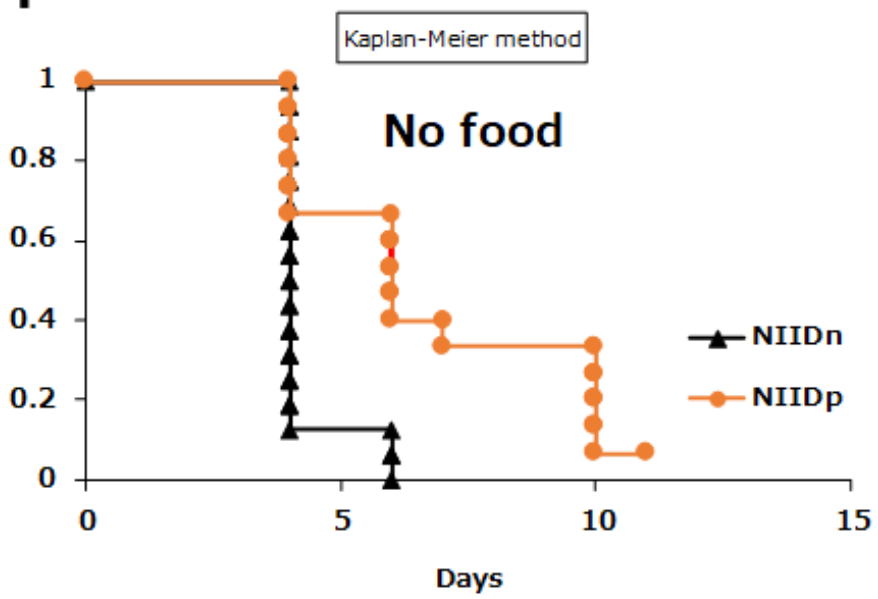

\section{Figure 1}

Survival rate of German cockroaches, Blattella germanica, under starvation conditions. Exp. 1: WATp cockroaches were infected with Blatticola blattae pinworms, and survival was compared with uninfected WATn cockroaches (WATp vs. WATn: $P=3.89 \times 10-8$ [log-rank test]). Exp. 2: NIIDp cockroaches were naturally infected with $B$. blattae, and the infection rate of NIIDn cockroaches was reduced by administration of the anthelmintic agent pyrvinium pamoate (NIIDp vs. NIIDn: P=0.048 [log-rank test]). 